\title{
Lyophilisate for Emulsion for Injection
}

National Cancer Institute

\section{Source}

National Cancer Institute. Lyophilisate for Emulsion for Injection. NCI Thesaurus. Code C158596.

Solid sterile preparation for veterinary use consisting of a freeze-dried powder intended to be mixed with the specified liquid to obtain an emulsion for injection. 